\title{
Salmon in pregnancy study (SIPS): the effects of increased oily fish intake during pregnancy on maternal peripheral blood mononuclear cell fatty acid composition and cytokine responses
}

\author{
L.-S. Kremmyda, M. Vlachava, P. S. Noakes, E. A. Miles, N. D. Diaper and P. C. Calder \\ Institute of Human Nutrition and DOHaD Division, School of Medicine, University of Southampton, IDS Building, \\ Southampton General Hospital, Southampton SO16 6YD, UK
}

The prevalence of childhood atopic diseases has increased during the last 30 years $^{(1)}$. Epidemiological studies have linked higher fish intake during pregnancy with lower risk of atopy in the offspring ${ }^{(2)}$. Furthermore, fish oil supplementation during pregnancy alters maternal and offspring immunity in a way that would be consistent with lowered risk of atopy ${ }^{(3)}$. Fish provide not only long chain (LC) $n-3$ polyunsaturated fatty acids (PUFAs) (eicosapentaenoic acid (EPA) and docosahexaenoic acid (DHA)) found in fish oils but also other nutrients. There are no studies of oily fish intervention in pregnancy and maternal immunity or infant atopy. The hypotheses examined by the work described here are that increased salmon consumption during pregnancy by women with high risk of having an atopic offspring will increase maternal peripheral blood mononuclear cell (PBMC) LC $n-3$ PUFA status and alter PBMC cytokine responses, which may potentially influence the developing foetal immune system in a way that would decrease atopy risk for the offspring.

Pregnant women $(n=123)$ with high risk of having atopic offspring and with low habitual intake of oily fish $(\leq 2 /$ month) were randomised at 20 weeks of pregnancy to either consuming two portions of farmed salmon a week or continuing their habitual diet until the end of their pregnancy. The women attended a clinic in the fasted state at weeks 20, 32-34 and 38 of pregnancy, at which blood samples were collected, and health and food frequency questionnaires were administered. Maternal PBMCs were isolated and their fatty acid composition was determined by gas chromatography. Cytokine responses by maternal PBMCs were measured after culturing with the mitogen phytohaemagglutinin for $48 \mathrm{~h}$; Interleukin (IL)-10, tumour necrosis factor (TNF)- $\alpha$ and interferon (IFN)- $\gamma$ concentrations were determined using the flow cytometry based cytometric bead array.

All 123 women attended the 20 week appointment, and 111 and 91 women attended the 34 and 38 week appointments, respectively. EPA, DHA and arachidonic acid in PBMCs declined during pregnancy in the control group $(P<0.001)$. In contrast, EPA was maintained in PBMCs in the salmon group $(P=0.876)$ and DHA increased $(P=0.027)$. PBMC EPA and DHA were higher in the salmon group compared to control at weeks 34 and 38 of pregnancy (both $P<0.001$ ). Production of IL-10, TNF- $\alpha$ and IFN- $\gamma$ was decreased throughout the course of pregnancy in both groups (all $P<0.001$ ). There were no significant differences between the groups, except that mitogeninduced IL-10 production was lower in the salmon group at week 34 of pregnancy $(P=0.045)$.

In conclusion, increased consumption of oily fish by pregnant women results in higher LC $n-3$ PUFA content of their immune cells, but this has limited impact on the function of those cells. The lower IL-10 production in the salmon group at 34 weeks of pregnancy may indicate an altered regulatory function.

This work was supported by the European Commission under Framework 6 and forms part of the AquaMax integrated project (FOOD-CT-2006-016249-2). The authors thank staff at the Princess Anne Hospital, Southampton, and the Medical Research Council Epidemiology Resource Centre for their invaluable contributions to this study.

1. Ninan TK \& Russell G (1992) BMJ 304, 873-875.

2. Willers S, Devereux G, Craig L et al. (2007) Thorax 62, 773-779.

3. Krauss-Etschmann S, Hartl D, Rzehak P et al. (2008) J Allergy Clin Immunol 121, 464-470. 